\title{
SMALL-SCALE FLUCTUATIONS AND ANISOTROPIES IN THE 1-3 KEV X-RAY BACKGROUND
}

\author{
X. Barcons \\ Department of Modern Physics \\ University of Cantabria \\ 39005 Santander Spain
}

\author{
A. C. Fabian \\ Institute of Astronomy \\ Madingley Road \\ Cambridge CB3 OHA UK
}

\begin{abstract}
The spatial distribution of the 1-3 keV X-ray background (XRB) in five Einstein Observatory Imaging Proportional Counter fields has been analyzed. The autocorrelation function does not exceed $9 \%$ on scales $-5^{\prime}$. The observed count probability distribution is then used to check the source number-flux distribution at faint levels. Agreement with the Einstein Observatory deep survey is obtained. A cutoff in the number-flux distribution for a Euclidean population of sources at a flux approximately one-half of the deep survey limit, previously suggested by Hamilton and Helfand (1987), is also inferred.
\end{abstract}

\section{INTRODUCTION}

Angular fluctuations and anisotropies of the XRB carry statistical information about the distribution of sources that give rise to its flux. In particular, the autocorrelation function (ACF) of the XRB is a measure of the integrated clustering amplitude of these sources, and fluctuations can be related to the flux distribution of the source population.

Here, we use five long-exposure fields obtained by the Einstein Observatory Imaging Proportional Counter (IPC) in the energy range 1-3 keV to study both the ACF of the XRB on small scales and the count probability distribution. More detailed information about the data and techniques used can be found in Barcons and Fabian (1989a; 1989b).

The 1-3 keV XRB flux is assumed to be the extrapolation, with an energy spectral index of 0.4 , of the well-measured flux at higher energies. Extra counts present in the IPC are associated with a homogeneous particle background. In this way, all fluctuations and anisotropies are assumed to be cosmic, in which case our results should be considered as upper limits to the structure of the XRB (see Hamilton and Helfand 1987).

\section{THE ANGULAR AUTOCORRELATION FUNCTION}

Counts falling in the central $0.25 \mathrm{deg}^{2}$ of the IPC (where the response is fairly constant) were grouped in $\sim 1 \operatorname{arcmin}^{2}$ pixels. Because the point spread function (PSF) has a greater extent than the pixel size, care has to be taken with statistical studies, since adjacent pixels will not be independent.

Bright sources were removed from any further analysis to minimize the effect of the PSF on the spatial anisotropies. A clearly positive signal in the ACF is seen on scales $\leq 4^{\prime}$, although it could be due mostly to the PSF of sources not detected.

A Gaussian ACF $\left(W(\theta)=W\left(5^{\prime}\right) \exp \left[\left(5^{\prime} / \theta_{o}\right)^{2}-\left(\theta / \theta_{o}\right)^{2}\right]\right)$, where $\theta_{o}$ is the correlation angle, was fitted to the data by minimizing the $\chi^{2}$ function. The best values are $\theta_{o}=4.1^{\prime}$ and $W\left(5^{\prime}\right)=0.05$ (Barcons and Fabian 1989a). An insight into the confidence levels for those 
parameters was achieved with a simple Monte Carlo simulation. At 95\% confidence level, $W\left(5^{\prime}\right) \leq 0.09$. Cosmological implications of this upper limit are being explored.

\section{THE COUNT PROBABILITY FUNCTION}

As shown by Scheuer (1974), the count probability function can be related to the $N(>S)$ curve (number of sources with flux greater than $S$ ), and the effective beam profile (convolution of the PSF with the pixel). This type of approach is most sensitive to the behavior of the $N(>S)$ curve at fluxes as faint as when there is about one source per beam (fainter sources producing essentially Gaussian noise).

Here, we take a pixel of $\sim 16 \operatorname{arcmin}^{2}$. As argued in the previous section, these pixels will be almost independent and therefore suitable for our analysis. The number-flux relation for sources is assumed to have a Euclidean shape $N(>S)=N_{o} S^{-1.5}$ with some minimum cutoff $S_{\min }$, which is related to the fraction $f$ of the extrapolated 1-3 keV XRB flux produced by this source population. The remaining flux is provided by a uniform background.

The normalization of $N(>S)$ is seen to be entirely consistent with the findings of the Einstein Observatory deep survey (Giacconi et al. 1979), in the sense that a surface density of about 18 sources $\mathrm{deg}^{-2}$ with fluxes greater than $S_{D S}=10^{-3}$ counts $\mathrm{s}^{-1}$ is inferred by minimizing the $\chi^{2}$ of the count probability function, with variable $N_{o}$.

Once $N_{o}$ is fixed, several values of $S_{\min }$ were tested. The best value of $S_{\min } \approx S_{D S} / 2$, in good agreement with previous work by Hamilton and Helfand (1987). In this case, sources produce a fraction $f \approx 35-45 \%$ (at $1 \sigma$ confidence). However, a Euclidean $N(>S$ ) extrapolated down to $S_{D S} / 15$ (where $f \approx 1$ ) can be only marginally excluded (less or about $3 \sigma$ level). The extent of the PSF does not allow us to set stronger constraints with existing data (see Barcons and Fabian $1989 b$ for details).

It must also be stressed that effects like source clustering and detector vignetting broaden the count probability function. If these effects are present (and they probably are), the values of $f$ given here are actually overestimates of its true value.

\section{CONCLUSION}

The 1-3 keV XRB maps a very uniform universe. A Euclidean population of sources is not adequate to produce the whole XRB. About 55-65\% of the XRB must then come either from a diffuse process or a new population of faint objects with a surface density exceeding a few thousands per square degree.

\section{REFERENCES}

Barcons, X. and Fabian, A. C. 1989a, MNRAS, 237, 119.

Barcons, X. and Fabian, A. C. $1989 b$, MNRAS, submitted.

Giacconi, R. et al. 1979, Ap. J., 234, L1.

Hamilton, T. T. and Helfand, D. J. 1987, Ap. J., 318, 93.

Scheuer, P. A. G. 1974, MNRAS, 166, 329. 